Research Paper

\title{
Nonlinear Stability Characteristics of Composite Cylindrical Panel Subjected to Non-uniform In-plane Mechanical and Localized Thermal Loadings
}

\author{
RAJESH KUMAR, L S RAMACHANDRA* and BISWANATH BANERJEE \\ Department of Civil Engineering, IIT Kharagpur 721 302, India
}

(Received on 20 April 2016; Accepted on 27 April 2016)

\begin{abstract}
The non-linear stability analysis of composite cylindrical panel subjected to concentrated in-plane mechanical and localized thermal loadings are reported here. The buckling of composite panel subjected to concentrated in-plane mechanical loading/ localized thermal loading is solved in two steps as the prebuckling stress distribution within the panel is not known a priori. In the first step, the semi-analytical expressions for the pre-buckling stresses within the composite cylindrical panel under in-plane mechanical/ thermal loadings are developed by solving in-plane elasticity/thermoelasticity problem. Subsequently, using these in-plane stresses within the cylindrical panel, the governing equations for nonlinear stability of layered composite panel are formulated using variational principle. The cylindrical panel is modeled based on Donnell's shallow shell theory considering higher order shear deformation theory and incorporating von-Kármán geometric nonlinearity. The Galerkin's method is used to solve the non-linear governing partial differential equations. The influence of different types of mechanical and thermal loadings, initial geometric imperfections, and radius of curvature on the postbuckling equilibrium paths is investigated.
\end{abstract}

Keywords: Composite Cylindrical Panel; Non-Uniform Mechanical Loading; Localized Thermal Loading; Galerkin Method; Nonlinear Stability

\section{Introdunction}

The classical buckling problem of composite cylindrical panel subjected to uniform in-plane mechanical loads or uniform thermal loading over plate area is well documented in literature. However, studies on buckling of composite panels subjected to concentrated inplane mechanical loading/localized thermal loading are meagre. Due to localized heating source at the centre of the panel or due to non-uniform exposure of the panel to thermal loading $T(x, y)$, the panel is subjected to localized thermal heating with maximum temperature at the centre and minimum temperature at the support. This has been idealized as (i) localized heating over central rectangular or circular region with constant temperature within the region or (ii) dome shaped heating $T(x, y)$. In the present article, the nonuniform mechanical loadings are modelled as concentrated load and partial edge load. Recent developments have shown that under the action of non-uniform mechanical in-plane loads, all three components of stresses $\left(\sigma_{x x}, \sigma_{y y}, \sigma_{x y}\right)$ are developed within the plate/panel. Thus, the buckling and post buckling problems of panel subjected to localized thermal loading or non-uniform mechanical loading involves two steps. In the first step, the prebuckling stress distributions within the panels are evaluated. Subsequently, using these stress distributions, the governing stability equations for panel are derived which are solved by Galerkin's method in the present article.

The numerous studies on the buckling and postbuckling response of composite flat panel under uniform temperature distribution over plan and through thickness are reported in the literature (Tauchert and Huang, 1987; Chen and Chen, 1989; Meyers and Hyer, 1991; Singh et al., 1993; Singha et

*Author for Correspondence: E-mail: lsr@civil.iitkgp.ernet.in 
al., 2001; Jones, 2005; Libtrescu et al. 2000, Ounis et $a l .$, 2014). Birman and Bert (1993) and Girish and Ramachandra (2006) traced the postbuckling equilibrium path of composite cylindrical shell panels subjected to combined mechanical and thermal loads (uniform temperature rise through the thickness) using one term and multi term Galerkin's method respectively. Yang et al. (2006) reported the postbuckling equilibrium path of functionally graded cylindrical panel with temperature dependent properties under thermomechanical loads within the framework of differential quadrature method. In the above papers, first, the panel was subjected to uniaxial mechanical load and then uniform temperature loading was applied. Dongyun et al. (2016) studied the postbuckling behaviour of stiffened curved panel experimentally and numerically (finite element method) under shear and in-plane mechanical loadings. The postbuckling response of composite cylindrical panel (Panda and Ramachandra, 2010) and sandwich panel (Dey and Ramachandra, 2014) under parabolically distributed edge loading was studied using multi term Galerkin's method. Authors used Ritz method for evaluating the in-plane stresses within curved panel due to non-uniform loading.

Chen et al. (1991) used finite element method to analyze the thermal buckling of composite flat panel under tent-like non-uniform temperature distribution. Mead (2003) used Rayleigh-Ritz method to analyze the buckling and free vibration of free-free isotropic flat panel under non-uniform in-plane thermal stresses. Author expressed the stress functions as orthonormal beam function and assumed that the flat panel is stressfree at all the edges to calculate in-plane stresses. Morimoto et al. (2006) evaluated the prebuckling thermal stress distribution within functionally graded rectangular plates due to localized thermals loadings by solving the thermoelastic problem. Using these inplane stresses the governing equations for the plate stability problem are formulated which are solved by Galerkin method to compute critical buckling temperature.

From the literature survey, it is observed that analytical expression is not available for prebuckling stress distribution within the layered composite cylindrical panel subjected to in-plane concentrated loading/localized thermal loading. In the present investigation, the semi-analytical expressions for the in-plane stresses within the layered composite cylindrical panel due to non-uniform in-plane mechanical / thermal loadings are developed by solving the in-plane elasticity problem. Subsequently, using these in-plane stresses within the panel, the governing nonlinear stability equations of layered composite cylindrical panel are formulated in terms of displacement $(u-v-w)$ and rotation $\left(\phi_{x}, \phi_{y}\right)$ variables. Using Galerkin's method, the governing partial differential equations satisfying the boundary conditions are reduced into a system of nonlinear algebraic equations. These equations are solved using Newton-Raphson method in conjunction with Riks approach to trace the postbuckling equilibrium paths. The effects of radius-to-width ratio, geometric imperfections and different types of non-uniform mechanical/localized thermal loadings on the nonlinear stability analysis of the layered composite cylindrical panel are examined.

\section{Formulation}

\section{Kinematic Equations}

Consider a composite cylindrical panel of length $a$, breadth $b$, thickness $h$ and composed of $N$ orthotropic layers of uniform thickness and subjected to nonuniform in-plane mechanical and thermal loading as shown in Fig. 1. Let the coordinate system of the middle surface of the panel coincide with $x-y$ axes of the cartesian co-ordinate $(x-y-\mathrm{z})$ system and the lines of principal curvature $(1 / R)$ of the middle surface, and $z$ coordinate is normal to the middle surface. In the present study the governing equation of the cylindrical panels is derived based on Donnell's shell theory considering higher order shear deformation theory. The strains at a distance $z$ from the middle surface can be expressed as (Soldatos, 1991),

$$
\left\{\begin{array}{l}
\varepsilon_{x x} \\
\varepsilon_{y y} \\
\gamma_{x y}
\end{array}\right\}=\left\{\begin{array}{l}
\varepsilon_{x x}^{o} \\
\varepsilon_{y y}^{o} \\
\gamma_{x y}^{o}
\end{array}\right\}+z\left\{\begin{array}{l}
\kappa_{x x} \\
\kappa_{y y} \\
\kappa_{x y}
\end{array}\right\}+f(z)\left\{\begin{array}{c}
\kappa_{x x}^{a} \\
\kappa_{y y}^{a} \\
\kappa_{x y}^{a}
\end{array}\right\}
$$

Where $\varepsilon_{x x}^{o}, \varepsilon_{y y}^{o}, \gamma_{x y}^{o}, \quad \kappa_{x x}, \kappa_{y y}, \kappa_{x y}$, $\kappa_{x x}^{a}, \kappa_{y y}^{a}, \kappa_{x y}^{a}$ and $\gamma_{y z}, \gamma_{x z}$ are membrane strains, bending strains, additional bending strains due to 
additional changes of curvature and shear strains respectively and can be defined as,

$$
\left\{\begin{array}{l}
\varepsilon_{x x}^{o} \\
\varepsilon_{y y}^{o} \\
\gamma_{x y}^{o}
\end{array}\right\}=\left\{\begin{array}{l}
u_{, x}^{o}+\frac{1}{2}\left(w_{, x}^{o}\right)^{2}+w_{, x}^{o} w_{, x}^{*} \\
v_{, y}^{o}+w / R+\frac{1}{2}\left(w_{, y}^{o}\right)^{2}+w_{, y}^{o} w_{, y}^{*} \\
u_{, y}^{o}+v_{, x}^{o}+w_{, x}^{o} w_{, y}^{o}+w_{, x}^{o} w_{, y}^{*}+w_{, y}^{o} w_{, x}^{*}
\end{array}\right\}
$$

$$
\left\{\begin{array}{l}
\kappa_{x x} \\
\kappa_{y y} \\
\kappa_{x y}
\end{array}\right\}=\left\{\begin{array}{l}
-w_{, x x}^{o} \\
-w_{, y y}^{o} \\
-2 w_{, y y}^{o}
\end{array}\right\}
$$

$$
\left\{\begin{array}{c}
\boldsymbol{\kappa}_{x x}^{a} \\
\boldsymbol{\kappa}_{y y}^{a} \\
\boldsymbol{\kappa}_{x y}^{a}
\end{array}\right\}=\left\{\begin{array}{l}
\phi_{x, x} \\
\phi_{y, y} \\
\phi_{x, y}+\phi_{y, x}
\end{array}\right\}
$$

$$
\left\{\begin{array}{l}
\gamma_{y z} \\
\gamma_{x z}
\end{array}\right\}=f^{\prime}(z)\left\{\begin{array}{l}
\phi_{y} \\
\phi_{x}
\end{array}\right\}
$$

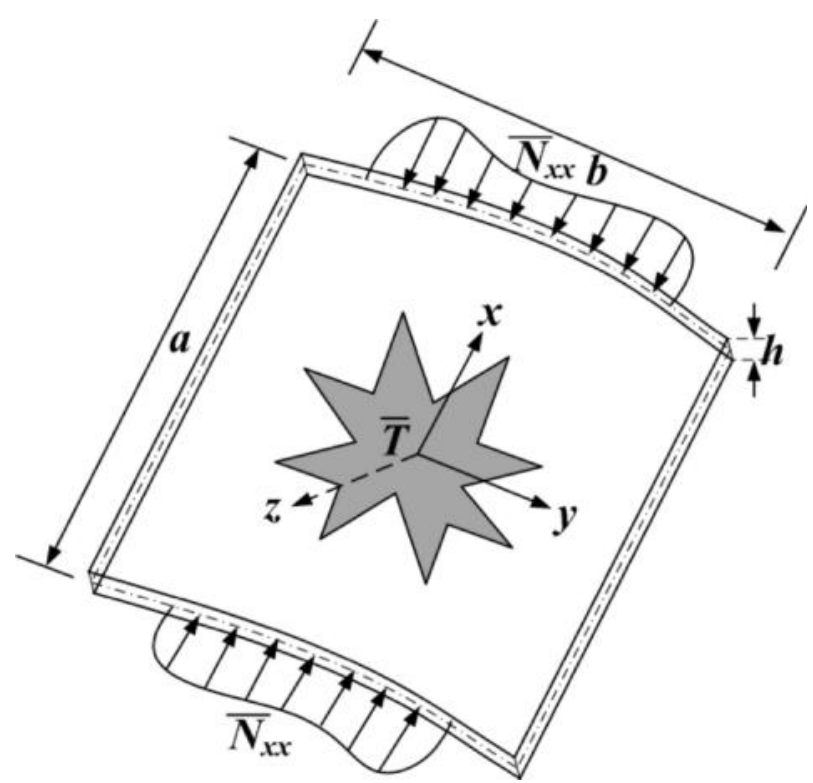

Fig. 1: Geometry and non-uniform mechanical and localized thermal loading for cylindrical panel
Here, $u, v$ and $w$ are the displacements at a generic point that is ' $z$ ' distance away from the middle surface of the panels along orthogonal co-ordinates $x, y$ and $z$, respectively. $u^{0}, v^{0}$ and $w^{0}$ the corresponding displacements of a generic point on the middle surface, and $\phi_{x}$ and $\phi_{y}$ are the rotation of cross sections initially perpendicular to the $x$ and $y$ axes, respectively. $(\cdot)_{, x}$ and $(\cdot)_{, y}$ denote the partial differentiation with respect to $x$ and $y$ respectively. In the equation (2), the von Kármán strain-displacement (nonlinear strains) relations are used to account for the deformed configuration of the shallow cylindrical panels with moderate displacements and small rotations. The initial geometric imperfection $w^{*}$ (i.e deviation of the middle surface from the perfect panel) is assumed as,

$$
w^{*}=e \cos \frac{\pi x}{a} \cos \frac{\pi y}{b}
$$

Where, $e$ is the amplitude of imperfection.

\section{Governing Differential Equations}

The partial differential equations governing the nonlinear stability of composite shallow cylindrical panel subjected to non-uniform in-plane mechanical and thermal loading in the cartesian co-ordinate system are expressed in terms of in-plane force, moment, additional moment and shear resultants respectively using variation principle as,

$$
\begin{array}{llllll}
\hat{N}_{x x, x}+\hat{N}_{x y, y} & 0 & & & \\
\hat{N}_{x y, x}+\hat{N}_{y y, y} & 0 & & & \\
-\left(\hat{N}_{y y} / R_{y}\right) & M_{x x, x x} & 2 M_{x y, x y} & M_{y y, y y} & & \\
+\left(\hat{N}_{x x} w,_{x}\right. & \left.\hat{N}_{x y} w,,_{y}\right),_{x} & \left(\hat{N}_{y y} w,,_{y}\right. & \left.\hat{N}_{x y} w,_{x}\right),_{y} & 0 & (7 \mathrm{a}-\mathrm{e}) \\
P_{x x, x}+P_{x y, y}-\mathrm{Q}_{x x}^{a} & 0 & & & & \\
P_{x y, x}+P_{y y, y}-\mathrm{Q}_{y y}^{a} & 0 & &
\end{array}
$$

Here,

Here, $\hat{N}_{i j}=N_{i j} \quad n_{i j} \quad\left(n_{T}\right)_{i j} \quad(i, j \quad x, y)$

In equation (8), $n_{i j}$ and $\left(n_{T}\right)_{i j}$ are the panel internal stress resultants due to non-uniform in-plane mechanical/localized thermal loadings respectively, and $N_{i j}$ are the secondary stress resultants due to the large deformation. Therefore, $\hat{N}_{i j}$ are the resultant cylindrical panel internal stress resultants. $M_{i j}$ and $P_{i j}$ 
are the moment and additional moment resultants respectively, and $Q_{x x}^{a}$ and $Q_{y y}^{a}$ are additional transverse shear force resultants.

The force, moment, additional moment and shear resultants are related to membrane, bending, additional bending and shear strains respectively, through the constitutive relations:

$$
\begin{aligned}
& \hat{N}=\mathbf{A} \bar{\varepsilon}^{\mathbf{0}}+\mathbf{B} \kappa+\mathbf{C} \kappa^{a}-N_{T} \\
& M=\mathbf{B}^{\mathbf{T}} \bar{\varepsilon}^{\mathbf{0}}+\mathbf{D} \kappa+\mathbf{E} \kappa^{a}-M_{T} \\
& M^{a}=\mathbf{C}^{\mathbf{T}} \bar{\varepsilon}^{\mathbf{0}}+\mathbf{E} \kappa+\mathbf{F} \kappa^{a}-M_{T}^{a} \\
& Q=\mathbf{H} \gamma
\end{aligned}
$$

Where, panel stiffness A, B, C, D, E, F and $\mathbf{H}$ are defined in terms of the transformed lamina stiffness $\overline{\mathbf{Q}}$ as,

$$
\begin{aligned}
& \left(A_{i j}, B_{i j}, D_{i j}\right)=\int_{-h / 2}^{h / 2} \bar{Q}_{i j}\left(1, z, z^{2}\right) d z \\
& =\sum_{k=1}^{N} \int_{z k-1}^{z_{k}} \bar{Q}_{i j}\left(1, z, z^{2}\right) d z(i, j=1,2,6) \\
& \left(C_{i j}, E_{i j}, F_{i j}\right)=\int_{-h / 2}^{h / 2} \bar{Q}_{i j}(1, z, f(z)) d z \\
& =\sum_{k=1}^{N} \int_{z_{k-1}}^{z_{k}} \bar{Q}_{i j}(1, z, f(z)) f(z) d z \quad(i, j=1,2,6) \\
& H_{i j}=\int_{-h / 2}^{h / 2} \bar{Q}_{i j} f^{\prime}(z) f^{\prime}(z) d z \\
& =\sum_{k=1}^{N} \int_{z_{k}}^{z_{k-1}} \bar{Q}_{i j} f^{\prime}(z) f^{\prime}(z) d z(i, j=4,5)
\end{aligned}
$$

Where, $N$ is the number of orthotropic layers. In the above equations (9)-(11), thermal force $\boldsymbol{N}_{T}^{T}=\left\{\begin{array}{lll}\left(N_{T}\right)_{x x} & \left(N_{T}\right)_{y y} & \left.\left(N_{T}\right)_{x y}\right\}, \quad \text { thermal }\end{array}\right.$ moment $\boldsymbol{M}_{T}^{T}=\left\{\begin{array}{lll}\left(M_{T}\right)_{x x} & \left(M_{T}\right)_{y y} & \left(M_{T}\right)_{x y}\end{array}\right\}$, and additional thermal moment $\boldsymbol{M}_{\boldsymbol{T}}^{a \mathrm{~T}}=$ $\left\{\left(M_{T}^{a}\right)_{x x}\left(M_{T}^{a}\right)_{y y}\left(M_{T}^{a}\right)_{x y}\right\}$ resultants are related to transformed thermal expansion coefficients $\boldsymbol{\alpha}^{\mathrm{T}}=\left\{\begin{array}{lll}\alpha_{x x} & \alpha_{y y} & \alpha_{x y}\end{array}\right\}$ by the relation,

$$
\begin{aligned}
& \left(\boldsymbol{N}_{\boldsymbol{T}}, \boldsymbol{M}_{\boldsymbol{T}}, \boldsymbol{M}_{\boldsymbol{T}}^{\boldsymbol{a}}\right) \\
& =\sum_{k=1}^{N} \int_{z_{k-1}}^{z_{k}} \overline{\mathbf{Q}} \boldsymbol{\alpha}(1, z, f(z)) T(x, y, z) d z
\end{aligned}
$$

Here, $\alpha_{x x}, \alpha_{y y}$ and $\alpha_{x y}$ are defined as,

$$
\begin{aligned}
& \alpha_{x x}=\alpha_{1} \cos ^{2} \theta+\alpha_{2} \sin ^{2} \theta \\
& \alpha_{y y}=\alpha_{1} \sin ^{2} \theta+\alpha_{2} \cos ^{2} \theta \\
& \alpha_{x y}=2\left(\alpha_{1}-\alpha_{2}\right) \sin \theta \cos \theta
\end{aligned}
$$

where, $\theta$ is the angle between $x$-axis and fiber direction. $\alpha_{1}$ and $\alpha_{2}$ are respectively the coefficients of thermal expansion in the principal material axes 1 and 2 . In the present case, the thermal moment and additional thermal moment resultants $\left(\boldsymbol{M}_{T}=\boldsymbol{M}_{T}^{a}=0\right)$ are zero due to uniform temperature rise though the panel thickness. It is also assumed that there is no heat exchange between the panel and the surroundings. Using equations (8)-(12), the non-linear governing partial differential equations of the layered composite cylindrical panel are expressed in terms of displacement $\left(u^{o}, v^{o}, w^{o}\right)$ and rotation $\left(\phi_{x}, \phi_{y}\right)$ variables, the details of which are given in Appendix A.

\section{In-Plane Elasticity Problem}

In this section, semi-analytical expression for the inplane pre-buckling stresses $\left(\sigma_{x x}, \sigma_{y y}\right.$ and $\left.\sigma_{x y}\right)$ within the layered composite shallow cylindrical panel under concentrated in-plane mechanical and localized thermal loadings are developed. For localized thermal loadings, the temperature distribution is assumed to be symmetrical about the co-ordinate axes and uniform across the thickness for ease of analytical development for the in-plane prebuckling stress expression within the cylindrical panel. Since the 
applied in-plane loading is not uniform, all three components of stresses exist within the composite cylindrical panel. These stresses have been computed by solving the plane elasticity problem and satisfying the in-plane stress boundary conditions for mechanical loads and in-plane displacement boundary conditions for localized thermal loadings.

The different distributions of in-plane mechanical loadings adopted in the present case along an edge of the panel are,

$\Re(y)=\frac{1}{c \sqrt{\pi}} \exp \left(-\frac{y^{2}}{c^{2}}\right)$ for concentrated load;

$=\frac{b}{d}\left(\frac{d}{b}+\sum_{n=1}^{\infty} \frac{2}{n \pi} \sin \frac{n \pi d}{b} \cos \frac{2 n \pi y}{b}\right)$ for partial load;

$=1$ for uniform load

Here, $c$ is the intensity of concentrated load whose value depends on numerical study and $d$ is the width of partial edge load.

The temperature distributions over the panel for different types of nonuniform heating are,

Full heating: $T(x, y)=T_{F}$

$\forall|x| \leq a / 2$ and $|y| \leq b / 2$

Dome profile heating:

$$
\begin{aligned}
& T(x, y)=T_{D} \cos (\pi x / a) \cos (\pi y / b) \\
& \forall|x| \leq a / 2 \text { and }|y| \leq b / 2
\end{aligned}
$$

Central circular heating:

$$
T(x, y)= \begin{cases}T_{C} & \text { at } \quad|y| \leq \sqrt{r^{2}-x^{2}} \\ & \text { and }|x| \leq r \\ 0 & \text { at } \sqrt{r^{2}-x^{2}}<|y| \leq b / 2 \\ & \text { and } r<|x| \leq a / 2\end{cases}
$$

Central rectangular heating:

$$
T(x, y)= \begin{cases}T_{R} & \text { at }|x| \leq a_{0} / 2 \\ & \text { and }|y| \leq b_{0} / 2 \\ 0 & \text { at } a_{0} / 2<|x| \leq a / 2 \\ & \text { and } b_{0} / 2<|y| \leq b / 2\end{cases}
$$

where, $T_{F}, T_{D}, T_{R}$ and $T_{C}$ are respectively the uniform temperature rise through its thickness for full heating, dome profile heating, central rectangular heating and central circular heating. $r$ is the radius of circular heating and, $a_{0}$ and $b_{0}$ are respectively the sides of the central rectangular heating area.

\section{(a) Stress distribution within the cylindrical panel under in-plane mechanical loading:}

Inplane stress equilibrium equation (in the absence of thermal loading) in terms of stress function $(\Phi)$ for composite cylindrical panel are obtained using straincompatibility conditions and are,

$a_{22} \frac{\partial^{4} \Phi}{\partial x^{4}}+\left(2 a_{12}+a_{66}\right) \frac{\partial^{4} \Phi}{\partial x^{2} \partial y^{2}}+a_{11} \frac{\partial^{4} \Phi}{\partial y^{4}}=0$

Here, the compatibility equation is written in terms of $x y$ coordinates instead of $R \theta$ coordinates as the shell panel is shallow. Airy's stress function $(\Phi)$ is defined by

$$
n_{x x}=\frac{\partial^{2} \Phi}{\partial y^{2}}, n_{y y}=\frac{\partial^{2} \Phi}{\partial x^{2}}, n_{x y}=-\frac{\partial^{2} \Phi}{\partial x \partial y}
$$

where, $a_{i j}$ is the flexibility coefficient of the laminated panel, and can be obtained using the following equations,

$$
\left[\begin{array}{lll}
a_{11} & a_{12} & a_{16} \\
a_{12} & a_{22} & a_{26} \\
a_{16} & a_{26} & a_{66}
\end{array}\right]=\left[\begin{array}{lll}
A_{11} & A_{12} & A_{16} \\
A_{12} & A_{22} & A_{26} \\
A_{16} & A_{26} & A_{66}
\end{array}\right]^{-1}
$$

Airy's stress solutions is assumed in terms of series as, 


$$
\begin{aligned}
\Phi(x, y) & =\sum_{i=1}^{\infty} r_{i}(y) \cos \left(\beta_{i} x\right) \\
& +\sum_{j=1}^{\infty} s_{j}(x) \cos \left(\theta_{j} y\right)+R_{0} y^{2}
\end{aligned}
$$

where, $\beta_{i}=\frac{2 i \pi}{a}, \theta_{j}=\frac{2 j \pi}{b}$

Substituting the above solutions in the inplane stress equilibrium equation (21) and equating the coefficients of $\cos \left(\beta_{i} x\right)$ and $\cos \left(\beta_{j} y\right)$ results in two ordinary differential equations in $r_{i}(y)$ and $s_{j}(x)$ respectively,

$$
\begin{aligned}
& a_{11} \frac{d^{4} r_{i}(y)}{d y^{4}}-\left(2 a_{12}+a_{66}\right) \beta_{i}{ }^{2} \frac{d^{2} r_{i}(y)}{d y^{2}}+a_{22} \beta_{i}{ }^{4} r_{i}(y)=0 \\
& a_{22} \frac{d^{4} s_{j}(x)}{d x^{4}}-\left(2 a_{12}+a_{66}\right) \theta_{j}{ }^{2} \frac{d^{2} s_{j}(x)}{d x^{2}}+a_{11} \theta_{j}{ }^{4} s_{j}(x)=0
\end{aligned}
$$

Substituting $r_{i}(y)=\exp (\lambda x)$ and $s_{j}(x)=\exp (\lambda x)$ in the above equations, the functions $r_{i}(y)$ and $s_{j}(x)$ are computed by considering the symmetry condition about $x$ and $y$ axes (i.e $r_{i}(y)=r_{i}(-y)$ and $s_{j}(x)=s_{j}(-$ $x)$. Thus,

$$
\begin{aligned}
& r_{i}(y)=R_{i 1} \cosh \left(\beta_{i 1} y\right)+R_{i 2} \cosh \left(\beta_{i 2} y\right) \\
& s_{j}(x)=S_{j 1} \cosh \left(\theta_{j 1} x\right)+S_{j 2} \cosh \left(\theta_{j 2} x\right)
\end{aligned}
$$

Substituting the expressions for $r_{i}(y)$ and $s_{j}(x)$ in equation (24), the expression for Airy's stress solution is written as,

$$
\begin{aligned}
\Phi(x, y) & =\sum_{i=1}^{\infty}\left\{\begin{array}{l}
R_{i 1} \cosh \left(\beta_{i 1} y\right)+ \\
R_{i 2} \cosh \left(\beta_{i 2} y\right)
\end{array}\right\} \cos \left(\beta_{i} x\right) \\
& +\sum_{j=1}^{\infty}\left\{\begin{array}{l}
S_{j 1} \cosh \left(\theta_{j 1} x\right)+ \\
S_{j 2} \cosh \left(\theta_{j 2} x\right)
\end{array}\right\} \cos \left(\theta_{j} y\right)+R_{0} y^{2}
\end{aligned}
$$

The inplane stress resultants are obtained by substituting the stress function (equation (24)) in equation (22). Thus,

$$
\begin{aligned}
n_{x x} & =\sum_{i=1}^{\infty} \cos \left(\beta_{i} x\right)\left(\begin{array}{l}
R_{i 1} \cosh \left(\beta_{i 1} y\right) \beta_{i 1}^{2} \\
+R_{i 2} \cosh \left(\beta_{i 2} y\right) \beta_{i 2}^{2}
\end{array}\right) \\
& -\sum_{j=1}^{\infty} \cos \left(\theta_{j} y\right)\left(\begin{array}{l}
S_{i 1} \cosh \left(\theta_{j 1} x\right) \\
+S_{i 2} \cosh \left(\theta_{j 2} x\right)
\end{array}\right) \theta_{j}{ }^{2}+2 R_{0}
\end{aligned}
$$

$$
\begin{aligned}
n_{y y} & =-\sum_{i=1}^{\infty} \cos \left(\beta_{i} x\right)\left(\begin{array}{l}
R_{i 1} \cosh \left(\beta_{i 1} y\right) \\
+R_{i 2} \cosh \left(\beta_{i 2} y\right)
\end{array}\right) \beta_{i}{ }^{2} \\
& +\sum_{j=1}^{\infty} \cos \left(\theta_{j} y\right)\left(\begin{array}{l}
S_{i 1} \cosh \left(\theta_{j 1} x\right) \theta_{j 1}^{2} \\
+S_{i 2} \cosh \left(\theta_{j 2} x\right) \theta_{j 2}^{2}
\end{array}\right) \\
n_{x y} & =\sum_{i=1}^{\infty} \sin \left(\beta_{i} x\right) \beta_{i}\left(\begin{array}{l}
R_{i 1} \sinh \left(\beta_{i 1} y\right) \beta_{i 1} \\
+R_{i 2} \sinh \left(\beta_{i 2} y\right) \beta_{i 2}
\end{array}\right) \\
& +\sum_{j=1}^{\infty} \sin \left(\theta_{j} y\right) \theta_{j}\left(\begin{array}{l}
S_{i 1} \sinh \left(\theta_{j 1} x\right) \theta_{j 1} \\
+S_{i 2} \sinh \left(\theta_{j 2} x\right) \theta_{j 2}
\end{array}\right)
\end{aligned}
$$

The coefficients, $R_{i 1}, R_{i 2}, S_{j 1}, S_{j 2}$ in expressions $n_{x x}(x, y), n_{y y}(x, y)$ and $n_{x y}(x, y)$ are determined using stress boundary conditions, which is defined as,

$$
\begin{aligned}
& n_{x x}\left( \pm \frac{a}{2}, y\right)=\Re(y), n_{x y}\left(x, \pm \frac{b}{2}\right)=0, \\
& n_{x y}\left( \pm \frac{a}{2}, y\right)=0, \quad n_{y y}\left(x, \pm \frac{b}{2}\right)=0
\end{aligned}
$$

Satisfying the boundary conditions results in simultaneous equations in terms of the unknown coefficients as,

$$
\begin{aligned}
& R_{i 1}=-\left(\frac{\beta_{i 2}}{\beta_{i 1}}\right) \frac{\sinh \frac{\beta_{i 2} b}{2}}{\sinh \frac{\beta_{i 1} b}{2}} R_{i 2} \\
& S_{j 1}=-\left(\frac{\theta_{j 2}}{\theta_{j 1}}\right) \frac{\sinh \frac{\theta_{j 2} a}{2}}{\sinh \frac{\theta_{j 1} a}{2}} S_{j 2}
\end{aligned}
$$




$$
\begin{aligned}
& \beta_{i}^{2}\left(\cosh \frac{\beta_{i 2} b}{2}-\frac{\beta_{i 2}}{\beta_{i 1}} \operatorname{coth} \frac{\beta_{i 1} b}{2} \sinh \frac{\beta_{i 2} b}{2}\right) R_{i 2} \\
& =-\left(\frac{2}{a}\right) \sum_{j=1}^{\infty} \frac{\theta_{j 2} \cos \frac{\theta_{j} b}{2}}{\sinh \frac{\theta_{j 1} a}{2}}\left(\begin{array}{l}
\theta_{j 1} \sinh \frac{\theta_{j 2} a}{2} I_{1} \\
-\theta_{j 2} \sinh \frac{\theta_{j 1} a}{2} I_{2}
\end{array}\right) S_{j 2} \\
& \theta_{j}^{2}\left(\cosh \frac{\theta_{j 2} a}{2}-\frac{\theta_{j 2}}{\theta_{j 1}} \operatorname{coth} \frac{\theta_{j 1} a}{2} \sinh \frac{\theta_{j 2} a}{2}\right) S_{j 2} \\
& +\left(\frac{2}{b}\right) I_{0}=-\left(\frac{2}{b}\right) \sum_{i=1}^{\infty} \frac{\beta_{i 2} \cos \frac{\beta_{i} a}{2}}{\sinh \frac{\beta_{i 1} b}{2}}\left(\begin{array}{l}
\beta_{i 1} \sinh \frac{\beta_{i 2} b}{2} I_{3} \\
-\beta_{i 2} \sinh \frac{\beta_{i 1} b}{2} I_{4}
\end{array}\right) R_{i 2} \\
& R_{0}=\frac{1}{b} \int_{0}^{b / 2} \Re(y) d y \\
& I_{1}=2 \int_{0}^{a / 2} \cosh \left(\theta_{j 1} x\right) \cos \left(\beta_{i} x\right) d x \\
& I_{2}=2 \int_{0}^{a / 2} \cosh \left(\theta_{j 2} x\right) \cos \left(\beta_{i} x\right) d x \\
& I_{3}=2 \int_{0}^{b / 2} \cosh \left(\beta_{i 1} y\right) \cos \left(\theta_{i} y\right) d y \\
& I_{4}=2 \int_{0}^{b / 2} \cosh \left(\beta_{i 2} y\right) \cos \left(\theta_{i} y\right) d y .
\end{aligned}
$$

$$
f_{i j}=\frac{16 T_{C}}{a b \theta_{j}} \int_{0}^{r}\left(\cos \left(\beta_{i} x\right) \sin \left(\theta_{j} \sqrt{r^{2}-x^{2}}\right)\right) d x
$$

for rectangular heating at the centre.

$$
f_{i j}=\frac{16 T_{R 2}}{a b \beta_{i} \theta_{j}} \sin \left(\beta_{i} a_{0} / 2\right) \sin \left(\theta_{j} b_{0} / 2\right)
$$

for rectangular heating at the centre.

$$
\text { and, } \beta_{i}=\frac{(2 i-1) \pi}{a}, \quad \theta_{j}=\frac{(2 j-1) \pi}{b} \text {. }
$$

Now, the thermal force resultants $N_{T}^{\mathrm{T}}=$ $\left\{\begin{array}{lll}\left(N_{T}\right)_{x x} & \left(N_{T}\right)_{y y} & \left(N_{T}\right)_{x y}\end{array}\right\}$ in equation (14) can be expressed in terms of in-plane temperature distribution as,

$$
N_{T}=N_{C} \sum_{i=1}^{\infty} \sum_{j=1}^{\infty} f_{i j} \cos \left(\beta_{i} x\right) \cos \left(\theta_{j} y\right)
$$

where, $\quad \boldsymbol{N}_{\boldsymbol{C}}^{\mathrm{T}}=\left\{\begin{array}{lll}\overline{N_{11}} & \overline{N_{21}} & \overline{N_{31}}\end{array}\right\}$ is termed as coefficient of thermal force resultants and is,

$$
\boldsymbol{N}_{C}=\sum_{k=1}^{N} \int_{z_{k-1}}^{z_{k}} \overline{\mathbf{Q}} \boldsymbol{\alpha} d z
$$

In-plane stress equilibrium equation (presence of thermal loading) in terms of Airy's stress function $(\Phi)$ is derived using strain-compatibility conditions and are defined as,

$$
\begin{aligned}
& a_{22} \frac{\partial^{4} \Phi}{\partial x^{4}}+\left(2 a_{12}+a_{66}\right) \frac{\partial^{4} \Phi}{\partial x^{2} \partial y^{2}}+a_{11} \frac{\partial^{4} \Phi}{\partial y^{4}} \\
& =a_{11} \frac{\partial^{2}\left(N_{T}\right)_{x x}}{\partial y^{2}}+a_{12}\left(\frac{\partial^{2}\left(N_{T}\right)_{x x}}{\partial x^{2}}+\frac{\partial^{2}\left(N_{T}\right)_{y y}}{\partial y^{2}}\right) \\
& +a_{22} \frac{\partial^{2}\left(N_{T}\right)_{y y}}{\partial x^{2}}-a_{66} \frac{\partial^{2}\left(N_{T}\right)_{x y}}{\partial x \partial y}
\end{aligned}
$$


In the above equation, $\left(N_{T}\right) x y$ is zero due to symmetric laminated composite cylindrical panel. The general solution $\boldsymbol{\Phi}(x, y)$ of equation (40) has two components: complementary solution $\Phi_{\mathrm{CS}}(x, y)$ and particular integral $\Phi_{\mathrm{PI}}(x, y)$,

$$
\Phi(x, y)=\Phi_{\mathrm{CS}}(x, y)+\Phi_{\mathrm{PI}}(x, y)
$$

Following Morimoto et al. (2006), the complementary solution is assumed in terms of series as,

$$
\begin{aligned}
\Phi_{\mathrm{CS}}(x, y) & =\sum_{i=1}^{\infty} r_{i}(y) \cos \left(\beta_{i} x\right) \\
& +\sum_{j=1}^{\infty} s_{j}(x) \cos \left(\theta_{j} y\right)
\end{aligned}
$$

Substituting the above complementary solutions in the in-plane stress equilibrium equation (40) and equating the coefficients of and results in two ordinary differential equations in and respectively,

$$
\begin{gathered}
a_{11} \frac{d^{4} r_{i}(y)}{d y^{4}}-\left(2 a_{12}+a_{66}\right) \beta_{i}{ }^{2} \frac{d^{2} r_{i}(y)}{d y^{2}} \\
+a_{22} \beta_{i}{ }^{4} r_{i}(y)=0 \\
a_{22} \frac{d^{4} s_{j}(x)}{d x^{4}}-\left(2 a_{12}+a_{66}\right) \theta_{j}{ }^{2} \frac{d^{2} s_{j}(x)}{d x^{2}} \\
+a_{11} \theta_{j}{ }^{4} s_{j}(x)=0
\end{gathered}
$$

Substituting $r_{i}(y)=\exp (\lambda y)$ and $s_{j}(x)=\exp (\lambda x)$ in the above equations, the functions and are computed by considering the symmetry about $x$ and $y$ axes (i.e., $r_{i}(y)=r_{i}(-y)$ and $s_{j}(x)=s_{j}(-x)$. Thus,

$$
\begin{aligned}
& r_{i}(y)=R_{i 1} \cosh \left(\beta_{i 1} y\right)+R_{i 2} \cosh \left(\beta_{i 2} y\right) \\
& s_{j}(x)=S_{j 1} \cosh \left(\theta_{j 1} x\right)+S_{j 2} \cosh \left(\theta_{j 2} x\right)
\end{aligned}
$$

Substituting the expressions for $r_{i}(y)$ and $s_{j}(x)$ in equation (42), the expression for complementary solution is written as,

$$
\begin{aligned}
\Phi_{\mathrm{CS}}(x, y) & =\sum_{i=1}^{\infty}\left\{\begin{array}{l}
R_{i 1} \cosh \left(\beta_{i 1} y\right) \\
+R_{i 2} \cosh \left(\beta_{i 2} y\right)
\end{array}\right\} \cos \left(\beta_{i} x\right) \\
& +\sum_{j=1}^{\infty}\left\{\begin{array}{l}
S_{j 1} \cosh \left(\theta_{j 1} x\right) \\
+S_{j 2} \cosh \left(\theta_{j 2} x\right)
\end{array}\right\} \cos \left(\theta_{j} y\right)
\end{aligned}
$$

The particular integral of equation (40) is written as,

$$
\begin{aligned}
& \Phi_{\mathrm{PI}}(x, y)=-\sum_{i=1}^{\infty} \sum_{j=1}^{\infty} f_{i j} \cos \left(\beta_{i} x\right) \cos \left(\theta_{j} y\right) \\
& \frac{\left(a_{12} \overline{N_{11}}+a_{22} \overline{N_{21}}\right) \beta_{i}{ }^{2}+\left(a_{11} \overline{N_{11}}+a_{12} \overline{N_{21}}\right) \theta_{j}{ }^{2}}{a_{22} \beta_{i}{ }^{4}+\left(2 a_{12}+a_{66}\right) \beta_{i}{ }^{2} \theta_{j}{ }^{2}+a_{11} \theta_{j}{ }^{4}}
\end{aligned}
$$

The in-plane thermal stress resultant due to localized thermal loadings is obtained by substituting the stress function (equation (41)) in equation (22). Thus,

$$
\begin{aligned}
& \left(n_{T}\right)_{x x}=\sum_{i=1}^{\infty} \cos \left(\beta_{i} x\right)\left(\begin{array}{l}
R_{i 1} \cosh \left(\beta_{i 1} y\right) \beta_{i 1}{ }^{2} \\
+R_{i 2} \cosh \left(\beta_{i 2} y\right) \beta_{i 2}{ }^{2}
\end{array}\right) \\
& -\sum_{j=1}^{\infty} \cos \left(\theta_{j} y\right)\left(\begin{array}{l}
S_{i 1} \cosh \left(\theta_{j 1} x\right) \\
+S_{i 2} \cosh \left(\theta_{j 2} x\right)
\end{array}\right) \theta_{j}^{2} \\
& +\sum_{i=1}^{\infty} \sum_{j=1}^{\infty} f_{i j} \cos \left(\beta_{i} x\right) \cos \left(\theta_{j} y\right) \theta_{j}{ }^{2} \\
& \frac{\left\{\left(a_{12} \overline{N_{11}}+a_{22} \overline{N_{21}}\right) \beta_{i}{ }^{2}+\left(a_{11} \overline{N_{11}}+a_{12} \overline{N_{21}}\right) \theta_{j}{ }^{2}\right\}}{a_{22} \beta_{i}{ }^{4}+\left(2 a_{12}+a_{66}\right) \beta_{i}{ }^{2} \theta_{j}{ }^{2}+a_{11} \theta_{j}{ }^{4}}
\end{aligned}
$$

$$
\begin{aligned}
\left(n_{T}\right)_{y y}= & -\sum_{i=1}^{\infty} \cos \left(\beta_{i} x\right)\left(\begin{array}{l}
R_{i 1} \cosh \left(\beta_{i 1} y\right) \\
+R_{i 2} \cosh \left(\beta_{i 2} y\right)
\end{array}\right) \beta_{i}{ }^{2} \\
& +\sum_{j=1}^{\infty} \cos \left(\theta_{j} y\right)\left(\begin{array}{l}
S_{i 1} \cosh \left(\theta_{j 1} x\right) \theta_{j 1}{ }^{2} \\
+S_{i 2} \cosh \left(\theta_{j 2} x\right) \theta_{j 2}{ }^{2}
\end{array}\right)
\end{aligned}
$$


$+\sum_{i=1}^{\infty} \sum_{j=1}^{\infty} f_{i j} \cos \left(\beta_{i} x\right) \cos \left(\theta_{j} y\right) \beta_{i}^{2}$

$\frac{\left\{\left(a_{12} \overline{N_{11}}+a_{22} \overline{N_{21}}\right) \beta_{i}{ }^{2}+\left(a_{11} \overline{N_{11}}+a_{12} \overline{N_{21}}\right) \theta_{j}{ }^{2}\right\}}{a_{22} \beta_{i}{ }^{4}+\left(2 a_{12}+a_{66}\right) \beta_{i}{ }^{2} \theta_{j}{ }^{2}+a_{11} \theta_{j}{ }^{4}}$

$$
\begin{aligned}
& \left(n_{T}\right)_{x y}=\sum_{i=1}^{\infty} \sin \left(\beta_{i} x\right) \beta_{i}\left(\begin{array}{l}
R_{i 1} \sinh \left(\beta_{i 1} y\right) \beta_{i 1} \\
+R_{i 2} \sinh \left(\beta_{i 2} y\right) \beta_{i 2}
\end{array}\right) \\
& +\sum_{j=1}^{\infty} \sin \left(\theta_{j} y\right) \theta_{j}\left(\begin{array}{l}
S_{i 1} \sinh \left(\theta_{j 1} x\right) \theta_{j 1} \\
+S_{i 2} \sinh \left(\theta_{j 2} x\right) \theta_{j 2}
\end{array}\right) \\
& +\sum_{i=1}^{\infty} \sum_{j=1}^{\infty} f_{i j} \sin \left(\beta_{i} x\right) \sin \left(\theta_{j} y\right) \beta_{i} \theta_{j} \\
& \frac{\left\{\left(a_{12} \overline{N_{11}}+a_{22} \overline{N_{21}}\right) \beta_{i}{ }^{2}+\left(a_{11} \overline{N_{11}}+a_{12} \overline{N_{21}}\right) \theta_{j}{ }^{2}\right\}}{a_{22} \beta_{i}{ }^{4}+\left(2 a_{12}+a_{66}\right) \beta_{i}{ }^{2} \theta_{j}{ }^{2}+a_{11} \theta_{j}{ }^{4}}
\end{aligned}
$$

For the case of thermal loading, the in-plane displacements are zero at the boundary. Therefore, in-plane displacements are obtained by substituting the in-plane thermal stress resultants $\left(\left(n_{T}\right)_{x x},\left(n_{T}\right)_{y y},\left(n_{T}\right)_{x y}\right)$ and thermal forces $\left(\left(N_{T}\right)_{x x},\left(N_{T}\right)_{y y},\left(N_{T}\right)_{x y}\right)$ in strains expression (equation (9)). Integrating strains expression, the inplane displacement are expressed as,

$u^{o}(x, y)=$

$-\sum_{i=1}^{\infty}\left[\begin{array}{l}R_{i 1} \sin \left(\beta_{i} x\right) \cosh \left(\beta_{i 1} y\right)\left(a_{11} \frac{\beta_{i 1}^{2}}{\beta_{i}}-a_{12} \beta_{i}\right) \\ +R_{i 2} \sin \left(\beta_{i} x\right) \cosh \left(\beta_{i 2} y\right)\left(a_{11} \frac{\beta_{i 2}^{2}}{\beta_{i}}-a_{12} \beta_{i}\right)\end{array}\right]$

$$
+\sum_{j=1}^{\infty}\left[\begin{array}{l}
S_{j 1} \sinh \left(\theta_{j 1} x\right) \cos \left(\theta_{j} y\right)\left(a_{11} \frac{\theta_{j}^{2}}{\theta_{j 1}}-a_{12} \theta_{j 1}\right) \\
+S_{j 2} \sinh \left(\theta_{j 2} x\right) \cos \left(\theta_{j} y\right)\left(a_{11} \frac{\theta_{j}^{2}}{\theta_{j 2}}-a_{12} \theta_{j 2}\right)
\end{array}\right]
$$$$
+\sum_{i=1}^{\infty} \sum_{j=1}^{\infty} f_{i j} \beta_{i} \sin \left(\beta_{i} x\right) \cos \left(\theta_{j} y\right)
$$$$
\left[\left(a_{11} a_{22}-a_{12}{ }^{2}\right) \overline{N_{11}} \beta_{i}{ }^{2}+a_{11} a_{66} \overline{N_{11}} \theta_{j}{ }^{2}\right.
$$$$
\frac{\left.+\left(a_{12}{ }^{2}-a_{11} a_{22}+a_{12} a_{66}\right) \overline{N_{21}} \theta_{j}{ }^{2}\right]}{a_{22} \beta_{i}^{4}+\left(2 a_{12}+a_{66}\right) \beta_{i}{ }^{2} \theta_{j}{ }^{2}+a_{11} \theta_{j}{ }^{4}}
$$

$v^{o}(x, y)=$

$\sum_{i=1}^{\infty}\left[\begin{array}{l}R_{i 1} \cos \left(\beta_{i} x\right) \sinh \left(\beta_{i 1} y\right)\left(a_{22} \frac{\beta_{i}^{2}}{\beta_{i 1}}-a_{12} \beta_{i 1}\right) \\ +R_{i 2} \cos \left(\beta_{i} x\right) \sinh \left(\beta_{i 2} y\right)\left(a_{22} \frac{\beta_{i}^{2}}{\beta_{i 2}}-a_{12} \beta_{i 2}\right)\end{array}\right]$

$-\sum_{j=1}^{\infty}\left[\begin{array}{c}S_{j 1} \cosh \left(\theta_{j 1} x\right) \sin \left(\theta_{j} y\right)\left(a_{22} \frac{\theta_{j 1}^{2}}{\theta_{j}}-a_{12} \theta_{j}\right) \\ +S_{j 2} \cosh \left(\theta_{j 2} x\right) \sin \left(\theta_{j} y\right)\left(a_{22} \frac{\theta_{j 2}^{2}}{\theta_{j}}-a_{12} \theta_{j}\right)\end{array}\right]$

$+\sum_{i=1}^{\infty} \sum_{j=1}^{\infty} f_{i j} \theta_{j} \cos \left(\beta_{i} x\right) \sin \left(\theta_{j} y\right)$

$\left[\left(a_{11} a_{22}-a_{12}{ }^{2}\right) \overline{N_{21}} \theta_{j}{ }^{2}+a_{22} a_{66} \overline{N_{21}} \beta_{i}{ }^{2}\right.$

$\frac{\left.+\left(a_{12}{ }^{2}-a_{11} a_{22}+a_{12} a_{66}\right) \overline{N_{11}} \beta_{i}{ }^{2}\right]}{a_{22} \beta_{i}{ }^{4}+\left(2 a_{12}+a_{66}\right) \beta_{i}{ }^{2} \theta_{j}{ }^{2}+a_{11} \theta_{j}{ }^{4}}$

The coefficients, $R_{i 1}, R_{i 2}, S_{j 1}, S_{j 2}$ in expression $u(x, y)$ and $v(x, y)$ are determined using immovable 
edge boundary conditions, $u^{0}=0, v^{0}=0$. Satisfying the immovable edge boundary conditions results in simultaneous equations in terms of the unknown coefficients as,

$$
\begin{aligned}
& R_{i 2}=-\frac{\cosh \frac{b \beta_{i 1}}{2}\left(a_{11} \frac{\beta_{i 1}{ }^{2}}{\beta_{i}}-a_{12} \beta_{i}\right)}{\cosh \frac{b \beta_{i 2}}{2}\left(a_{11} \frac{\beta_{i 2}{ }^{2}}{\beta_{i}}-a_{12} \beta_{i}\right)} R_{i 1} \\
& S_{j 2}=-\frac{\cosh \frac{a \theta_{j 1}}{2}\left(a_{22} \frac{\theta_{j 1}^{2}}{\theta_{j}}-a_{12} \theta_{j}\right)}{\cosh \frac{a \theta_{j 2}}{2}\left(a_{22} \frac{\theta_{j 2}{ }^{2}}{\theta_{j}}-a_{12} \theta_{j}\right)} S_{j 1} \\
& -\frac{4}{b}\left(a_{11} \frac{\beta_{i 1}^{2}}{\beta_{i}}-a_{12} \beta_{i}\right) \sin \frac{a \beta_{i 1}}{2}
\end{aligned}
$$

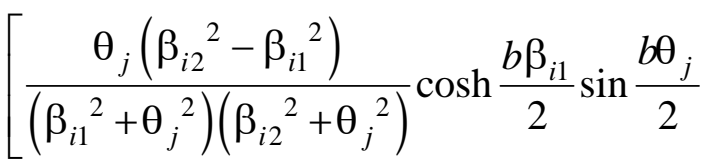

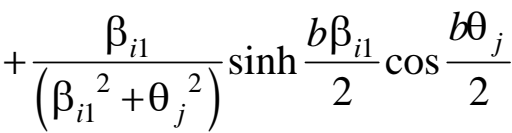$$
\left.-\frac{\beta_{i 2}}{\left({\left.\beta_{i 2}{ }^{2}+\theta_{j}{ }^{2}\right)}_{)}\right.} \frac{\cosh \frac{b \beta_{i 1}}{2}}{\cosh \frac{b \beta_{i 2}}{2}} \sinh \frac{b \beta_{i 2}}{2} \cos \frac{b \theta_{j}}{2}\right] R_{i 1}
$$$$
+\left[\left(a_{11} \frac{\theta_{j}^{2}}{\theta_{j 1}}-a_{12} \theta_{j 1}\right) \sinh \frac{a \theta_{j 1}}{2}-\left(a_{11} \frac{\theta_{j}^{2}}{\theta_{j 2}}-a_{12} \theta_{j 2}\right)\right.
$$$$
\left.\frac{\left(a_{22} \frac{\theta_{j 1}{ }^{2}}{\theta_{j}}-a_{12} \theta_{j}\right)}{\left(a_{22} \frac{\theta_{j 2}^{2}}{\theta_{j}}-a_{12} \theta_{j}\right)} \frac{\cosh \frac{a \theta_{j 1}}{2}}{\cosh \frac{a \theta_{j 2}}{2}} \sinh \frac{a \theta_{j 2}}{2}\right] S_{j 1}
$$

$$
\begin{aligned}
& \left.\frac{\cosh \frac{b \beta_{i 1}}{2}}{\cosh \frac{b \beta_{i 2}}{2}} \sinh \frac{b \beta_{i 2}}{2}\right] R_{i 1} \\
& -\frac{4}{a}\left(a_{22} \frac{\theta_{j 1}^{2}}{\theta_{j}}-a_{12} \theta_{j}\right) \sin \frac{b \theta_{j}}{2}
\end{aligned}
$$

$$
\left[\frac{\beta_{i}\left(\theta_{j 2}{ }^{2}-\theta_{j 1}^{2}\right)}{\left(\theta_{j 1}{ }^{2}+\beta_{i}^{2}\right)\left(\theta_{j 2}{ }^{2}+\beta_{i}^{2}\right)} \cosh \frac{a \theta_{j 1}}{2} \sin \frac{a \beta_{i}}{2}\right.
$$$$
+\frac{\theta_{j 1}}{\left(\theta_{j 1}^{2}+\beta_{i}^{2}\right)} \sinh \frac{a \theta_{j 1}}{2} \cos \frac{a \beta_{i}}{2}
$$

$$
\left.-\frac{\theta_{j 2}}{\left(\theta_{j 2}{ }^{2}+\beta_{i}^{2}\right)} \frac{\cosh \frac{a \theta_{j 1}}{2}}{\cosh \frac{a \theta_{j 2}}{2}} \sinh \frac{a \theta_{j 2}}{2} \cos \frac{a \beta_{i}}{2}\right] S_{j 1}
$$

$+\sum_{j=1}^{\infty} f_{i j} \theta_{j} \sin \frac{b \theta_{j}}{2}$ 


$$
\begin{aligned}
& {\left[\left(a_{11} a_{22}-a_{12}{ }^{2}\right) \overline{N_{21}} \theta_{j}{ }^{2}+a_{22} a_{66} \overline{N_{21}} \beta_{i}{ }^{2}\right.} \\
& \frac{\left.+\left(a_{12}{ }^{2}-a_{11} a_{22}+a_{12} a_{66}\right) \overline{N_{11}} \beta_{i}{ }^{2}\right]}{a_{22} \beta_{i}{ }^{4}+\left(2 a_{12}+a_{66}\right) \beta_{i}{ }^{2} \theta_{j}{ }^{2}+a_{11} \theta_{j}{ }^{4}}=0
\end{aligned}
$$

Solving the above simultaneous equations, the unknown coefficients, $R_{i 1}, R_{i 2}, S_{j 1}, S_{j 2}$ are determined. Now, the explicit expression for thermal stress distribution of layered composite panel under nonuniform thermal loading are computed using equations (47)-(49). Subsequently, suing these stress distributions, the governing equations for shallow shell panel stability are formulated in displacement and rotation variables which are given in Appendix A.

\section{Solution Technique}

\section{Boundary Conditions of the Problem}

In the present investigation, simply supported boundary conditions are considered in the out of plane direction of the cylindrical panel. The movable edge in-plane boundary conditions are assumed for mechanical loads and immovable edge in-lane boundary conditions are assumed for thermal loading. The following two sets of simply supported boundary conditions are assumed for cylindrical panel,

Movable edge boundary conditions:

$$
\begin{array}{r}
N_{x x}=v^{o}=w^{o}=P_{x x}=\phi_{y}^{o}=M_{x x}=0 \\
\text { at } \quad x=-a / 2, a / 2 \\
u^{o}=N_{y y}=w^{o}=\phi_{x}^{o}=P_{y y}=M_{y y}=0 \\
\text { at } \quad y=-b / 2, b / 2
\end{array}
$$

Immovable edge boundary conditions:

$$
\begin{array}{r}
u^{o}=v^{o}=w^{o}=P_{x x}=\phi_{y}^{o}=M_{x x}=0 \\
\text { at } \quad x=-a / 2, a / 2 \\
u^{o}=v^{o}=w^{o}=\phi_{x}^{o}=P_{y y}=M_{y y}=0 \\
\text { at } y=-b / 2, b / 2
\end{array}
$$

To satisfy the above simply supported boundary conditions, the following sets of displacement fields are assumed in the Galerkin's method.

Displacement fields for movable edge boundary conditions are,

$$
\begin{aligned}
& \tilde{u}^{o}=\sum_{m=1}^{i} \sum_{n=1}^{j} U_{m n} \sin \left(\frac{m \pi x}{a}\right) \cos \left(\frac{n \pi y}{b}\right) \\
& \tilde{v}^{o}=\sum_{m=1}^{i} \sum_{n=1}^{j} V_{m n} \cos \left(\frac{m \pi x}{a}\right) \sin \left(\frac{n \pi y}{b}\right) \\
& \tilde{w}^{o}=\sum_{m=1}^{i} \sum_{n=1}^{j} W_{m n} \cos \left(\frac{m \pi x}{a}\right) \cos \left(\frac{n \pi y}{b}\right) \\
& \tilde{\phi}_{x}=\sum_{m=1}^{i} \sum_{n=1}^{j} K_{m n} \sin \left(\frac{m \pi x}{a}\right) \cos \left(\frac{n \pi y}{b}\right) \\
& \tilde{\phi}_{y}=\sum_{m=1}^{i} \sum_{n=1}^{j} K_{m n} \cos \left(\frac{m \pi x}{a}\right) \sin \left(\frac{n \pi y}{b}\right)
\end{aligned}
$$

Displacement fields for immovable edge boundary conditions are,

$$
\begin{aligned}
& \tilde{u}^{o}=\sum_{m=1}^{i} \sum_{n=1}^{j} U_{m n} \sin \left(\frac{2 m \pi x}{a}\right) \cos \left(\frac{n \pi y}{b}\right) \\
& \tilde{v}^{o}=\sum_{m=1}^{i} \sum_{n=1}^{j} V_{m n} \cos \left(\frac{m \pi x}{a}\right) \sin \left(\frac{2 n \pi y}{b}\right) \\
& \tilde{w}^{o}=\sum_{m=1}^{i} \sum_{n=1}^{j} W_{m n} \cos \left(\frac{m \pi x}{a}\right) \cos \left(\frac{n \pi y}{b}\right) \\
& \tilde{\phi}_{x}=\sum_{m=1}^{i} \sum_{n=1}^{j} K_{m n} \sin \left(\frac{2 m \pi x}{a}\right) \cos \left(\frac{n \pi y}{b}\right) \\
& \tilde{\phi_{y}}=\sum_{m=1}^{i} \sum_{n=1}^{j} K_{m n} \cos \left(\frac{m \pi x}{a}\right) \sin \left(\frac{2 n \pi y}{b}\right)
\end{aligned}
$$

Where, $m$ and $n$ denote the number of half waves in $x$ and $y$ directions. The resulting algebraic equations after the Galerkin projection of the nonlinear governing equations are solved using NewtonRaphson method in conjunction with Riks approach to trace the post-buckling equilibrium path.

\section{Results and Discussions}

To validate the present formulation, the postbuckling equilibrium paths of layered composite cylindrical panel under uniform uni-axial compressive loading and full thermal loading over the panel are compared with literature and ABAQUS results in Fig. 2a and Fig. $2 b$. It is observed from these figures that the postbuckling equilibrium path obtained for in-plane mechanical loading compares well with that of Girish and Ramachandra (2006) and the equilibrium path 


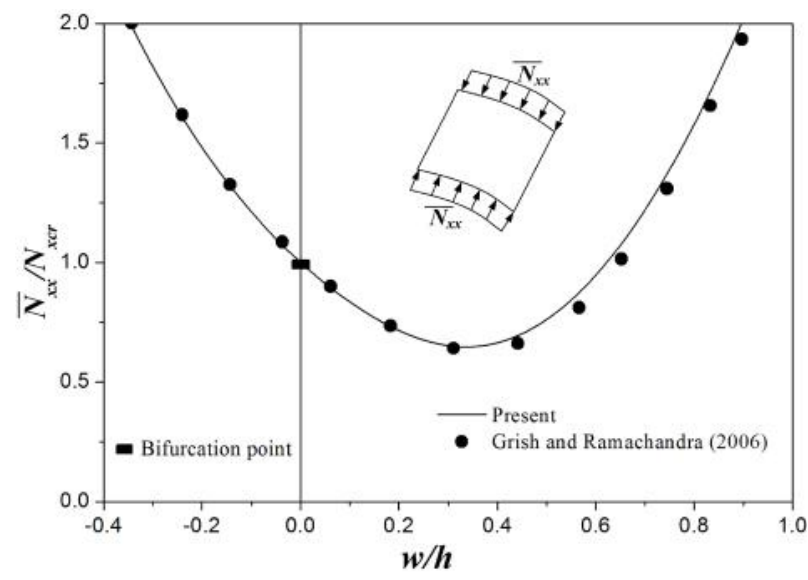

Fig. 2A: Postbuckled equilibrium paths of a three layered symmetric cross-ply [0/90//0] simply supported composite cylindrical panel $(a / b=1, a / h=50, R / a=$ 5) under uniform uni-axial in-plane loading

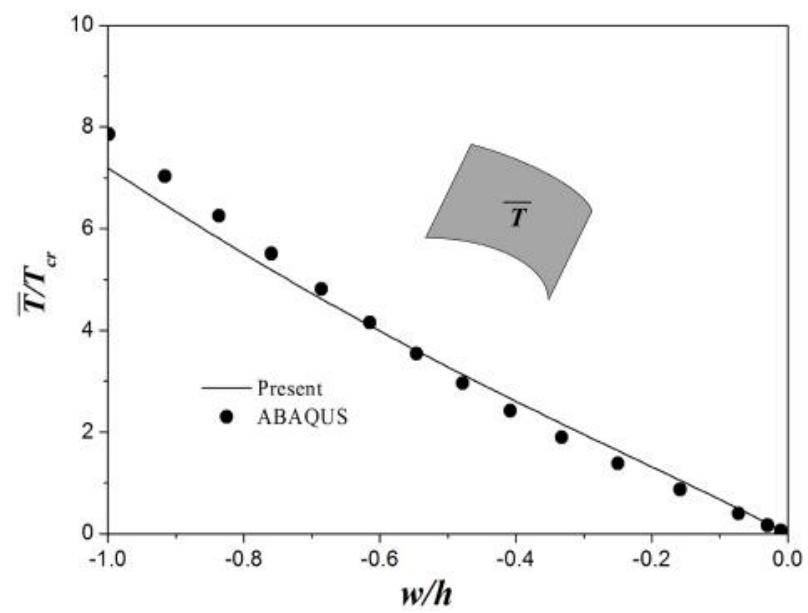

Fig. 2B: Postbuckled equilibrium path of a four layered symmetric cross-ply [0/90/90/0] simply supported 10) under full thermal loading

$a / b=1, b / h=100, R / b=$

for thermal loading compares fairly well with that of ABAQUS results. Here, $N_{\text {xcr }}$ and $T_{\text {cr }}$ are the critical buckling load and of a shallow panel and critical temperature of a flat panel respectively.

Following material properties are used for validating the postbuckling equilibrium path of cylindrical panel under in-plane mechanical / thermal loading.

After validating the formulation, some new results for postbuckling equilibrium paths of composite cylindrical panel subjected to different types of nonuniform in-plane mechanical and thermal loadings are presented in this section.
Following material properties are used in the present study:

$$
\text { (i) } \begin{aligned}
& E_{11} / E_{22}=25, G_{12}=G_{13}=0.5 E_{22}, \\
& \alpha_{1}=0.1 \times 10^{-5}, v_{12}=0.25,
\end{aligned}
$$

for

tracing the postbuckling equilibrium paths of cylindrical panel under in-plane mechanical loading.

$$
\text { (ii) } \begin{aligned}
& E_{11} / E_{22}=20, G_{12}=G_{13}=0.5 E_{2}, \\
& \alpha_{1}=0.1 \times 10^{-5}, v_{12}=0.25,
\end{aligned}
$$

for

tracing the postbuckling equilibrium paths of cylindrical panel under thermal loading.

The postbuckling equilibrium paths of the cylindrical panel are traced for three types of in-plane mechanical loadings: concentrated, partial and uniform load. To trace the thermal postbuckling equilibrium paths, two sets of thermal loadings have been considered. In the first set, four cases of the partial central rectangular heating have been considered. The length and width of heating region for four cases are,

Case 1: $a_{0} / b=b_{0} / b=0.25$,

Case 2: $a_{0} / b=b_{0} / b=0.50$,

Case 3: $a_{0} / b=b_{0} / b=0.75$,

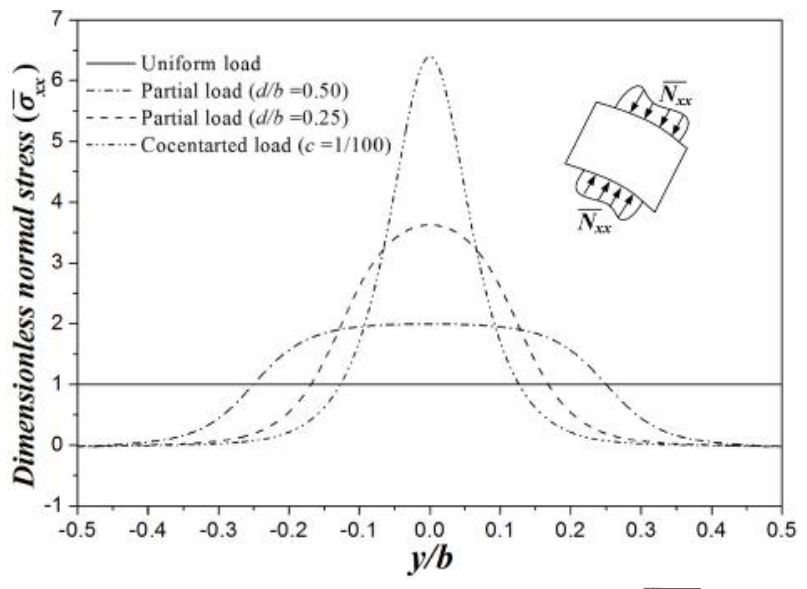

Fig. 3A: The dimensionless stress distribution $\left(c_{x x}\right)$ for a composite panel along line $x=0$ for different nonuniform in-plane mechanical loadings 


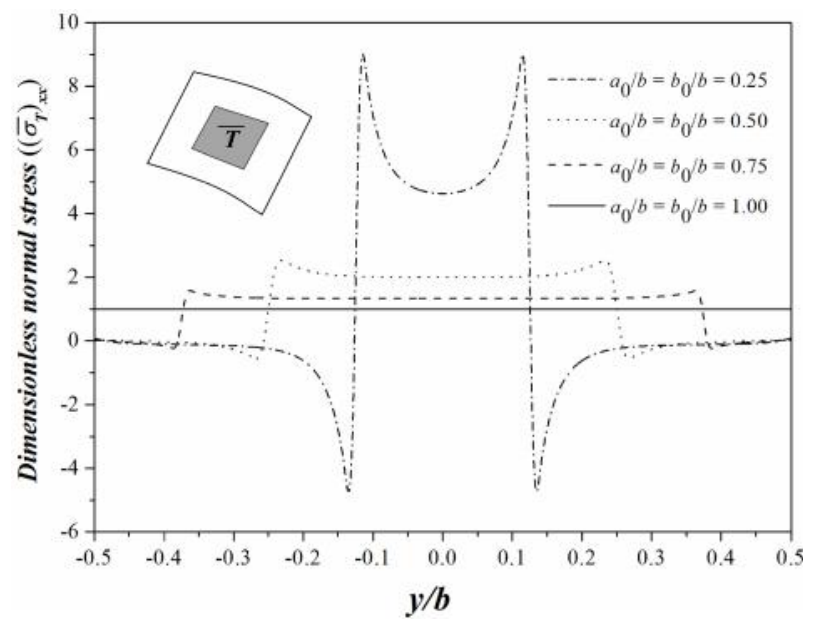

Fig. 3B: The dimensionless in-plane thermal normal stress $\left(\left(\bar{\sigma}_{T}\right)_{x x}=\left(\sigma_{T}\right)_{x x} /\left(\sigma_{T}\right)_{x x}^{F}\right)$ distribution for composite panel along line $\mathrm{x}=\mathbf{0}$ for four cases of partial central rectangular heating

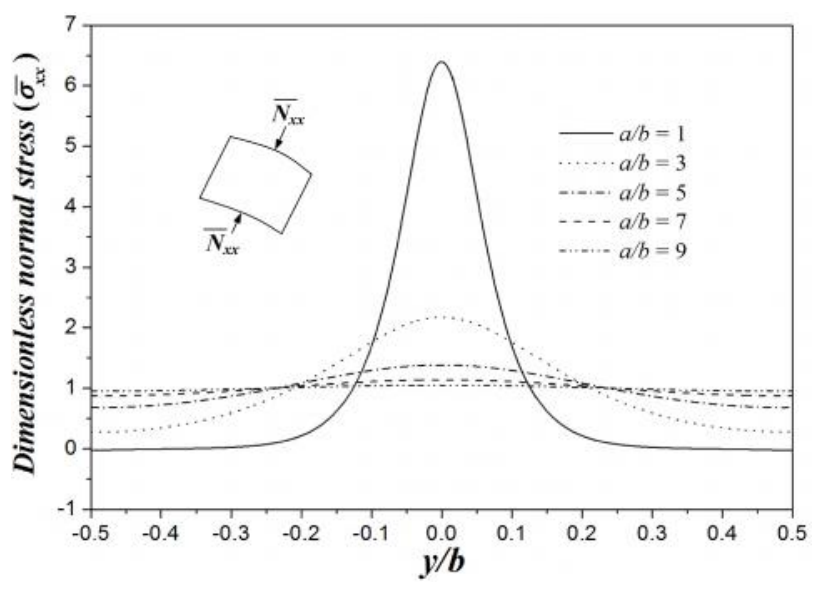

Fig. 3C: The dimensionless in-plane stress $\left(\sigma_{x x}\right)$ distribution for a composite panel along line $x=0$ under concentrated in-plane mechanical loading for different aspect ratios

Case 4: $a_{0} / b=b_{0} / b=1.00$.

In the above four cases, width of the panel $(b)$ is kept constant and the area of heating region $\left(A_{0}=a_{0} \times b_{0}\right)$ is varied.

In the second set, four different types of thermal heating have been considered over square plate. They are full heating, dome profile heating, central circular heating and central rectangular heating. The radius of circular heating $(r)$ is taken as $\frac{b}{2 \sqrt{2}}$ and side for rectangular heating is $a_{0} / b=0.50$ and $b_{0} / b=0.50$.

Fig. 3A and Fig. 3B, show the dimensionless inplane normal stress distribution for a four layered cross-ply [0/90/90/0] shallow cylindrical composite panel $(a / b=1, a / h=100)$ under different types of nonuniform mechanical in-plane loadings and four cases partial central rectangular heating respectively. The normal stresses due to nonuniform mechanical loading/localized heating are made dimensionless by dividing it by stresses corresponding to uniform inplane mechanical loading/full heating. The distributions of loadings are only different but the total load applied is kept the same for all types of mechanical loadings while calculating the prebuckling stress distribution. The total heat supplied for each case of central rectangular heating is the same. Therefore, the uniform temperature rise through the panel thickness for four cases can be related by,

$$
\frac{T_{R 1}}{16}=\frac{T_{R 2}}{4}=\frac{9 T_{R 3}}{16}=\frac{T_{R 4}}{1}
$$

where, $T_{R 1}, T_{R 2}, T_{R 3}$ and $T_{R 4}$ are temperature rise through the panel thickness for case 1, case 2, case 3 and case 4 respectively.

As per St. Venant's principle, the applied loading must diffuse into a state of uniform stress away from the boundary. It is well known that the stress distribution becomes uniform for the case of uniform mechanical loading and full thermal heating for square composite panel. However, the prebuckling stress distribution become uniform only for panel with higher aspect ratio $(a / b \geq 9)$ under nonuniform mechanical in-plane loading (Fig. 3C).

The postbuckling equilibrium paths are represented as a plot of dimensionless in-plane load $\left(\bar{N}_{x x} / N_{x c r}\right.$ or $\left.\bar{T} / T_{c r}\right)$ against dimensionless out-ofplane displacement $(w / h)$ at the centre. In Fig. 4 , the postbuckling equilibrium paths for simply supported four layered symmetric cross-ply [0/90/90/0] composite cylindrical panel $(a / b=1, b / h=100, R / b=$ 10) subjected to concentrated in-plane loading are traced considering different number of terms in the displacement fields. The converged result for 
equilibrium path is obtained for displacement field with $u, v, w, \phi_{x}$ and $\phi_{y}$. The subsequent results are reported with four terms in the displacement fields. In Fig. 5, the postbuckling equilibrium paths of simply supported four layered symmetric cross-ply [0/90/90/0] composite cylindrical panel $(a / b=1, b / h=100, R / b=10)$ is given for various types of inplane loading distribution. Here, $N_{\text {xcr }}$ is the critical load of panel under concentrated loading. Bifurcation buckling is observed for panels under uniform loading only. Bifurcation point is not observed for other loading cases as non-uniform loading induces compressive stress as well tensile stress within the panel. The tensile stress in the unloading direction causes the panel to deflect out-of-plane as soon as the load is applied due to curvature of the panel. In all cases snap through buckling is observed.

In Fig. 6, the postbuckling equilibrium paths of simply supported four layered symmetric cross-ply [0/90/90/0] composite cylindrical panel $(a / b=1, b / h$ $=100)$ subjected to concentrated inplane loading is given for different radius to width $(R / b)$ ratios. It is observed from the figure that the limit point and snap through behaviour exist for panels with $R / b \leq 10$. For higher radius to width ratios the postbuckled equilibrium path shows hardening type of behaviour. The influence of initial geometric imperfections on the postbuckling equilibrium path of a simply supported four layered symmetric cross-ply [0/90/90/0] composite cylindrical panel $(a / b=1, b / h=100, R / b=$ 10) under concentrated in-plane loading is presented in Fig. 7.

It is observed from the figure that the bifurcation buckling does not occur in case of perfect panels under concentrated in-plane mechanical loading. Snap through behaviour is observed due to initial inward (+ve) imperfections $(0.01 h, 0.05 h, 0.1 h)$ and for small magnitude of outward (-ve) imperfection $(-0.01 h)$. The panel deflects outward for higher amplitude of outward imperfections $(-0.05 h,-0.1 h)$ and shows hardening behaviour.

The influence of load ratio $\left(N_{0}= \pm \bar{N}_{y y} / \bar{N}_{x x}\right)$ on the postbuckling equilibrium path of a simply supported four layered symmetric cross-ply [0/90/90/0] composite cylindrical panel (a/

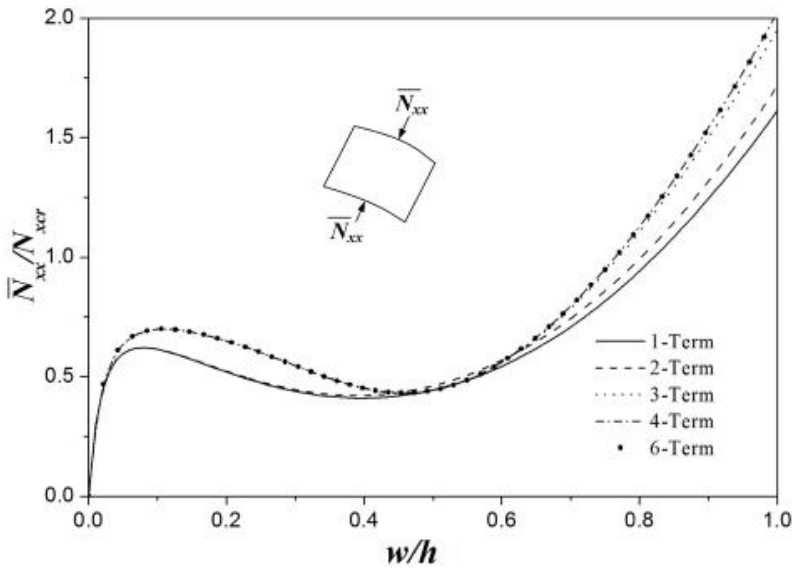

Fig. 4: Postbuckled equilibrium paths of a simply supported composite cylindrical panel under concentrated inplane loading

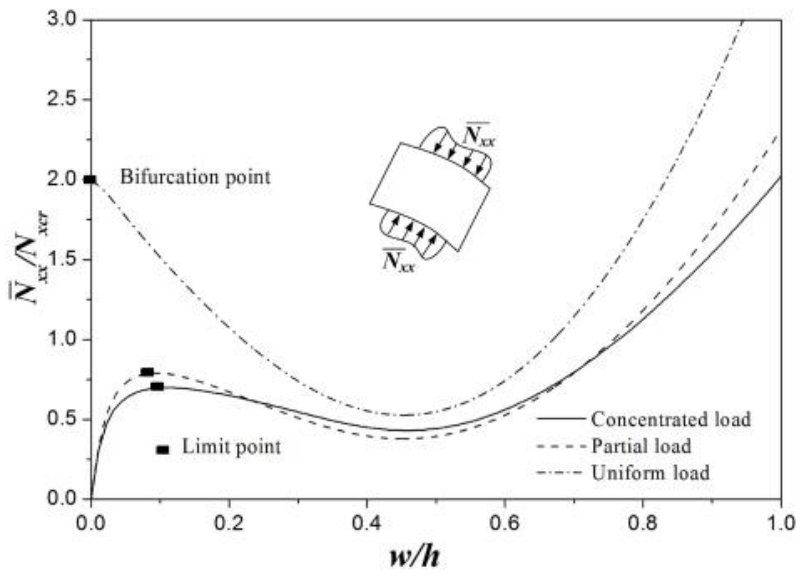

Fig. 5: The effect of different types of nonuniform in-plane loadings on the equilibrium path of a simply supported composite cylindrical panel

$b=1, b / h=100, R / b=10)$ under concentrated inplane loading is presented in Fig. 8. The load ratio is defined as the ratio of compressive/tensile edge load in the $y$-direction to compressive edge load in the $x$ direction. Here, compressive loading is taken as positive and tensile in-plane loading is taken as negative. It is observed from the curves that the panel deflects in the inward direction for all negative load ratios. Also, for small positive value of load ratio $\left(N_{0}\right.$ $=0.01,0.015)$ the panel deflect in the inward direction and exhibit snap though behaviour, and for higher value of load ratio $\left(N_{0}=0.025\right)$ the panel deflects in the outward direction and shows hardening behaviour. After limit point the load carrying capacity of shell panels (Figs. 6, 7 and 8) initially decrease due to decrease in curvature of the deformed panel. After 


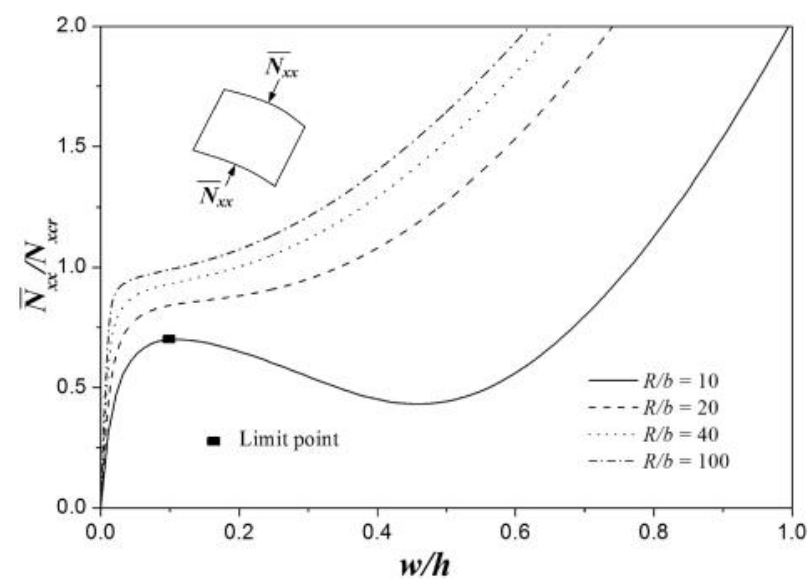

Fig. 6: The influence of radius-to-width $(R / b)$ ratio on the equilibrium path of a simply supported composite cylindrical panel under concentrated in-plane loading

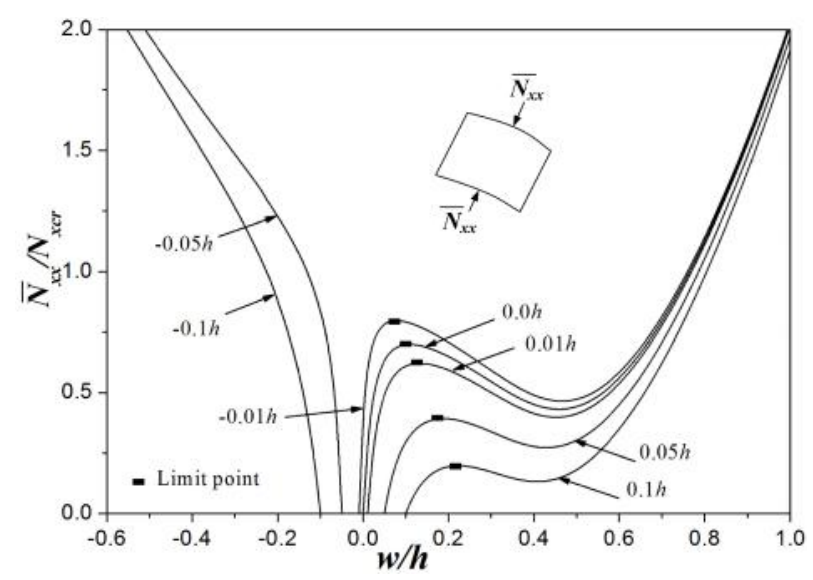

Fig. 7: The influence of geometric imperfections on the postbuckling equilibrium path of a simply supported composite cylindrical panel under concentrated inplane loading

flattening of shell panel, it develops curvature in the opposite direction which results in increased load carrying capacity.

Fig. 9 shows the postbuckling equilibrium path of a four layered cross-ply [0/90/90/0] simply supported composite cylindrical panel $(a / b=1, b / h=$ $100, R / b=10$ ) under four cases of partial central rectangular heating. In the figure, $T_{\mathrm{cr}}$ is the critical load of the flat panel under full heating. Due to thermal loadings the biaxial compressive stresses are developed within the heating region which causes the panel to deflect outwards and hence curvature of the panel is increased which results in hardening behaviour. The temperature deflection curve is almost

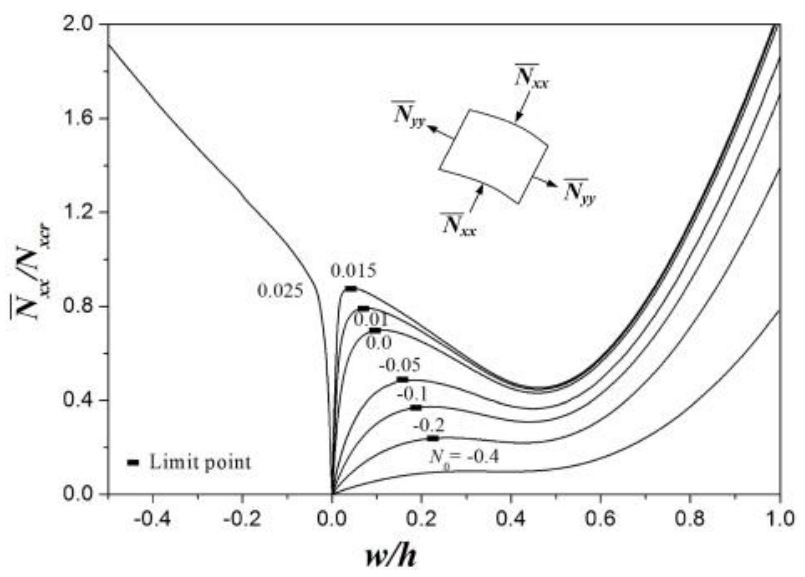

Fig. 8: The influence of load ratio $\left(\mathrm{N}_{0}\right)$ on the postbuckling equilibrium path of a simply supported composite cylindrical panel under concentrated in-plane loading

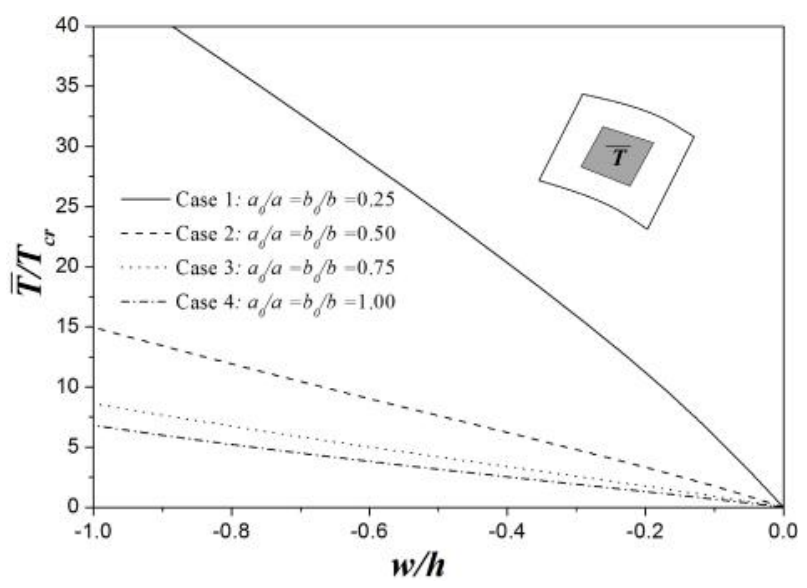

Fig. 9: Influence of four cases of the localized thermal loading on the equilibrium path of a simply supported composite cylindrical panel

linear for the case of full heating and it becomes nonlinear for localized heating.

Fig. 10 shows the postbuckling equilibrium path of a four layered cross-ply [0/90/90/0] simply supported composite cylindrical panel $(a / b=1, b / h=$ $100, R / b=10)$ under different types of thermal heating. Here, $T_{c r}$ is the critical load of the flat panel under full heating. It is observed from the figure that the panel deflects in the outward direction due to compressive stress within heating region in both the directions. This results in hardening type of behaviour. It is observed from the figure that the panel deflects in the outward direction for all the cases of localized thermal loadings. 


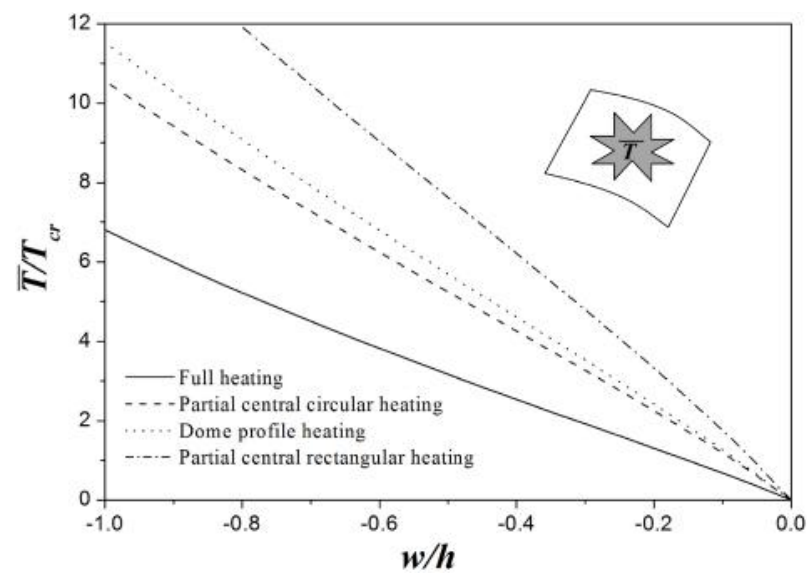

Fig. 10: The influence of different types of nonuniform heating on the postbuckling equilibrium path of a simply supported composite cylindrical panel

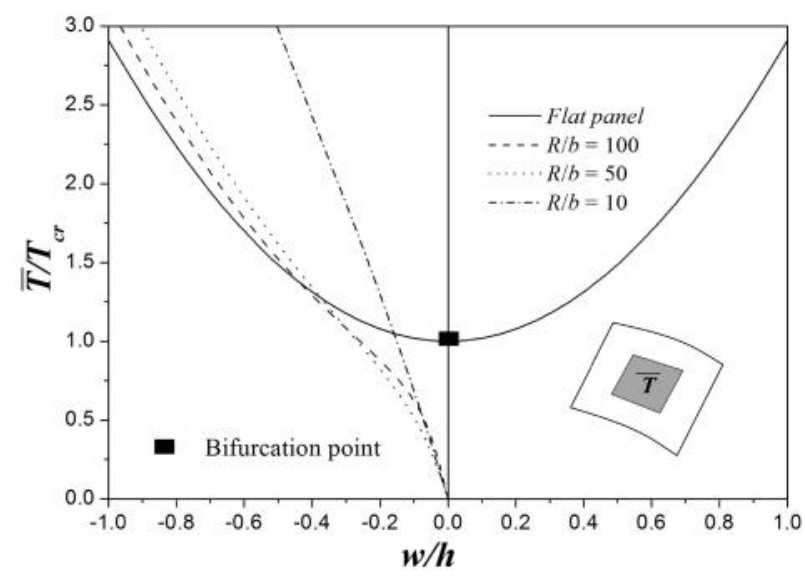

Fig. 11: The influence of radius-to-width ratio on the equilibrium path of a simply supported composite cylindrical panel under localized $\left(a_{0} / b=b_{0} / b=0.50\right)$ central rectangular heating

The influence of radius to width ratios on the postbuckling equilibrium path of a simply supported four layered symmetric cross-ply [0/90/90/0] composite cylindrical panel $(a / b=1, b / h=100)$ under partial central rectangular heating $\left(a_{0} / b=b_{0} / b=0.50\right)$ is presented in Fig. 11. Here, $T_{c r}$ is the critical load of the flat. It is observed from the figure that the panel deflects in the outward direction due to biaxial compressive stresses within heating region. The equilibrium path of the flat panel is symmetrical as shown in the figure under localized thermal loading. Hardening behaviour of composite panel increases with decrease of radius to width ratios.

\section{Conclusions}

In this article for the first time, semi-analytical expressions for the in-plane stresses within the layered composite cylindrical panel due to non-uniform inplane mechanical/localized thermal loadings are developed by solving the in-plane elasticity/ thermoelasticity problem. Subsequently, using these prebuckling stress distributions, the governing nonlinear stability equations of panel are derived. Panels are modelled based on Donnell's shallow shell theory considering higher order shear deformation theory and incorporating von-Kármán geometric nonlinearity. These are solved using Galerkin method.

It is observed that for nonuniform in-plane mechanical loading the prebuckling stresses become uniform only for higher aspect ratio. The localized thermal heating induces all three components prebuckling stresses within the panel. In case of localized heating, both and are compressive within the heating region and stresses suddenly drop and become tensile outside the heating region and they gradually become zero at the boundary. The magnitude of tensile stress decreases with the increase of localized heating area. Whereas in case of nonuniform mechanical loading is tensile at some locations of the panel and there is no sharp change in the magnitude of stress as in the case of localized thermal loading. Bifurcation buckling is not observed in case panels subjected to non-uniform mechanical loadings due to tensile stresses developed in the unloaded direction. The tensile stress in the unloaded direction causes the panel to deflect out-of-plane as soon as the load is applied due to curvature of the panel. Under thermal loadings the panel exhibit hardening type of equilibrium paths due to in-plane stress distribution within the panel. Due to biaxial compression under thermal loading, the panel initially deflects outwards (-ve direction) and hence curvature of the panel is increased which results in hardening behaviour. Under concentrated loading, snap through behaviour is observed due to initial inward imperfections and for small magnitude of outward imperfection. The panel deflects outward for higher amplitude of outward imperfections and shows hardening type of behaviour. The deformation of the panel is sensitive to the type of mechanical load applied and magnitude and direction of initial imperfections. 


\section{Appendix A}

Non-linear governing partial differential equations of symmetric cross-ply composite cylindrical panel in terms of displacement $\left(u^{0}, v^{0}, w^{0}\right)$ and rotation

$\left(\phi_{x}, y\right)$ variables are given below:

$$
\begin{aligned}
& A_{11} u_{, x x}^{o}+A_{66} u_{, y y}^{o} \quad\left(\begin{array}{ll}
A_{12} & \left.A_{66}\right) v_{, x y}^{o}
\end{array}\right. \\
& +\left\{A_{11}\left(w_{, x x}^{o} \quad w_{, x x}^{*}\right) \quad A_{66}\left(w_{, y y}^{o} \quad w_{, y y}^{*}\right)\right\} w_{, x}^{o} \\
& +\left(A_{11} w_{, x x}^{o} \quad A_{66} w_{, y y}^{o}\right) w_{, x}^{*} \quad\left(\begin{array}{ll}
A_{12} & A_{66}
\end{array}\right) \\
& \left(w_{, y}^{o} w_{, x y}^{o}+w_{, y}^{o} w_{, x y}^{*} \quad w_{, y}^{*} w_{, x y}^{o}\right) \\
& \begin{array}{llll}
-n_{x x, x}-\left(n_{T}\right)_{x x, x} & n_{x y, y} & \left(n_{T}\right)_{x y, y} & 0
\end{array} \\
& \left(A_{12}+A_{66}\right) u_{, x y}^{o} \quad A_{66} v_{, x x}^{o} \quad A_{22} v_{, y y}^{o} \\
& +\left\{A_{66}\left(w_{, x x}^{o} \quad w_{, x x}^{*}\right) \quad A_{22}\left(w_{, y y}^{o} \quad w_{, y y}^{*}\right)\right\} w_{, y}^{o} \\
& +\left(A_{66} w_{, x x}^{o} \quad A_{22} w_{, y y}^{o}\right) w_{, y}^{*} \quad\left(\begin{array}{ll}
A_{12} & A_{66}
\end{array}\right) \\
& \left(w_{, x}^{o} w_{, x y}^{o}+w_{, x}^{o} w_{, x y}^{*} \quad w_{, x}^{*} w_{, x y}^{o}\right) \\
& \begin{array}{llll}
-n_{x y, x}-\left(n_{T}\right)_{x y, x} & n_{y y, y} & \left(n_{T}\right)_{y y, y} & 0
\end{array}
\end{aligned}
$$

\section{References}

Birman V and Bert C W (1993) Buckling and post-buckling of composite plates and shells subjected to elevated temperature J Applied Mechanics ASME 60 514-519

Chen L W and Chen L Y (1989) Thermal postbuckling analysis of laminated composite plates by the finite element method Composite Structuture 12 257-270

Chen W J, Lin P D and Chen W (1991) Thermal buckling behavior of thick composite laminated plates under non-uniform temperature distributions Composite Structuture 41637 645

Dey T and Ramachandra L S (2014) "Buckling and postbuckling response of sandwich panels under non-uniform mechanical edge loadings Composite Part B 60 537-545

Dongyun Ge, Yuming Mo, Boling He, Yiting Wu and Xuzhen Du

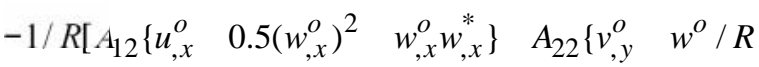

$$
\begin{aligned}
& \left.\left.+0.5\left(w_{, y}^{o}\right)^{2} \quad w_{, y}^{o} w_{, y}^{*}\right\} \quad n_{y y} \quad\left(n_{T}\right)_{y y}\right] \\
& -\left\{D_{11} w_{, x x x x}^{o} \quad 2\left(D_{12} \quad 2 D_{66}\right) w_{, x x y y}^{o} \quad D_{22} w_{, y y y y}^{o}\right\} \\
& +E_{11} a_{x, x x x} \quad\left(E_{12} \quad 2 E_{66}\right)_{x, x y y} \quad E_{22} y, y y y \\
& +\left(E_{12}+2 E_{66}\right) \emptyset_{y, x x y} \quad E_{11} x, x x x \quad\left(\begin{array}{ll}
E_{12} & 2 E_{66}
\end{array}\right) \\
& \left(\mathscr{o}_{x, x y y}+\mathscr{o}_{y, x x y}\right) \quad E_{22} y, y y y \quad\left[A _ { 1 1 } \left(u_{, x}^{o} \quad 0.5\left(w_{, x}^{o}\right)^{2}\right.\right. \\
& \left.+w_{, x}^{o} w_{, x}^{*}\right) \quad A_{12}\left(v_{, y}^{o} \quad w^{o} / R \quad 0.5\left(w_{, y}^{o}\right)^{2} \quad w_{, y}^{o} w_{, y}^{*}\right)
\end{aligned}
$$

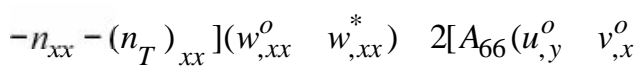

$$
\begin{aligned}
& \left.\left.\left.+w_{, x}^{o} w_{, y}^{o} \quad w_{, x}^{o} w_{, y}^{*} \quad w_{, x}^{*} w_{, y}^{o}\right) \quad n_{x y} \quad\left(n_{T}\right)_{x y}\right\}\right]\left(\begin{array}{ll}
w_{, x y}^{o} & \left.w_{, x y}^{*}\right)
\end{array}\right.
\end{aligned}
$$

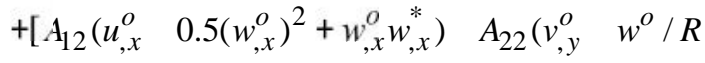

$$
\begin{aligned}
& \left.\left.+0.5\left(w_{, y}^{o}\right)^{2} \quad w_{, y}^{o} w_{, y}^{*}\right) \quad n_{y y} \quad\left(n_{T}\right)_{y y}\right]\left(w_{, y y}^{o} \quad w_{, y y}^{*}\right) \quad 0 \\
& -E_{11} w_{, x x x}^{o} \quad\left(E_{12} \quad 2 E_{66}\right) w_{, x y y}^{o} \\
& +F_{11} b_{x, x x} \quad F_{66} x, y y \quad\left(F_{12} \quad F_{66}\right)_{y, x y} \\
& -H_{55} a_{x} \quad 0 \\
& -E_{22} w_{, y y y}^{o} \quad\left(E_{12} \quad 2 E_{66}\right) w_{, x x y}^{o} \\
& +F_{66} C_{y, x x} \quad F_{22} \quad y, y y \quad\left(F_{12} \quad F_{66}\right)_{x, x y} \\
& -H_{44} C_{y} \quad 0
\end{aligned}
$$

(2016) Experimental and numerical investigation of stiffened composite curved panel under shear and in-plane bending Composite Structuture 137 185-195

Girish J and Ramachandra L S (2006) Thermomechanical postbuckling analysis of cross-ply laminated cylindrical shell panels J Engineering Mechanics 132 133-140

Jones R M (2005) Thermal buckling of uniformly heated unidirectional and symmetric cross-ply laminated fiberreinforced composite uniaxial in-plane restrained simply supported rectangular plates Composite Part A 36 13551367

Librescu L, Nemeth M P, Starnes J H and Lin W (2000) Nonlinear response of flat and curved panels subjected to thermomechanical loads Journal of Thermal Stresses 23549582

Mead D J (2003) Vibration and buckling of flat free-free plates 
under non-uniform in-plane thermal stresses Journal of Sound and Vibration 260 141-165

Meyers C A and Hyer M W (1991) Thermal buckling and postbuckling of symmetrically laminated composite plates Journal of Thermal Stresses 14 519-540

Morimoto T, Tanigawa Y and Kawamura R (2006) Thermal buckling of functionally graded rectangular plates subjected to partial heating International Journal of Mechanical Sciences $\mathbf{4 8}$ 926-937

Ounis H, Tati A and Benchabane A (2014) Thermal buckling behavior of laminated composite plates: A finite-element study Frontiers of Mechanical Engineering 9 41-49

Panda S K and Ramachandra L S (2010) Postbuckling analysis of cross-ply laminated cylindrical shell panels under parabolic mechanical edge loading Thin-Walled Structure 48 660667
Singha M K, Ramachandra L S and Bandyopadhyay J N (2001) Stability and strength of composite skew plates under thermomechanical loads AIAA Journal 39 1618-1623

Singh G, Rao G V and Iyengar N G R (1993) Thermal postbuckling behavior of rectangular antisymmetric cross-ply composite plates Acta Mechanica 98 39-50

Soldatos K P 1991 A refined laminated plate and shell theory with Journal of Sound and Vibration 144 109-129

Tauchert T R and Huang N N (1987) Thermal buckling of symmetric angle-ply laminated plates IH Marshall (editor) Composite Structuture 4 (4th ICCS) Paisley, Scotland. London: Elsevier 1.424-1.435

Yang J, Liew K M, Wu Y F and Kitipornchai S (2006) Thermomechanical post-buckling of FGM cylindrical panels with temperature-dependent properties International Journal of Solids and Structures 43 307-324. 